\title{
Statistical Analysis of Fluctuation Characteristics at High- and Low-Field Sides in L-mode SOL Plasmas of JT-60U
}

\author{
N. Ohno1), H. Tanaka1), N. Asakura2), Y. Tsuji1), S. Takamura3), Y. Uesugi4) and JT-60U \\ Team2) \\ 1)Graduate School of Engineering, Nagoya Univ. Nagoya, Aichi-ken 464-8603, Japan \\ 2)Japan Atomic Energy Agency, Naka, Ibaraki-ken 311-0193, Japan \\ 3)Department of Electronics, Faculty of Engineering, Aichi Institute of Technology, \\ Yachikusa 1247, Yakusa-cho, Toyota 470-0392, Japan \\ 4)Faculty of Engineering, Kanazawa University, 2-40-20 Kodatsuno, Ishikawa-ken 920-8667, Japan \\ e-mail contact of main author: ohno@ees.nagoya-u.ac.jp
}

\begin{abstract}
Detailed comparison between fluctuation characteristics at high-field-side (HFS) and low-fieldside (LFS) scrape off layers (SOLs) has been made, for the first time, in the L mode plasma of JT-60U tokamak using reciprocating Langumuir probes. Statistical analysis based on probability distribution function (PDF) was employed to describe intermittent (non-diffusion) transport in SOL plasma fluctuations. It was found that the positive bursty events appeared most frequently at LFS midplane associated with blobby plasma transport, then the PDF is strongly skewed positively, while the PDF in HFS SOL is close to Gaussian distribution. Conditional averaging analysis of the positive bursty events at LFS midplane indicates the intermittent feature with a rapid increase and a slow decay is similar to that of plasma blobs theoretically predicted. Statistical self-similarity was also investigated with Fourier power spectrum, and statistics of waiting-time and duration-time of the fluctuation. It was found, for the first time, that clear statistical self-similarity was observed at LFS SOL, showing fractal property of the fluctuation. The scaling exponent of duration time statistics disagrees with the predictions for the self-organized criticality (SOC) paradigm.
\end{abstract}

\section{Introduction}

Intermittent convective plasma transport, so-called "plasma blobs" has been observed in low-field-side (LFS) scrape off layers (SOLs) of several tokamak devices[1-8], which is thought to play a key role for cross-field transport. It has strong influence on recycling processes and impurity generation from a first wall. Theory predicts that plasma blobs move toward first wall at LFS due to $\boldsymbol{E} \times \boldsymbol{B}$ drift, where the charge separation in a plasma blob is driven by gradient and curvature of magnetic field[9]. Then, it is expected that the property of blobby plasma transport is quite different in high-field-side (HFS) SOLs in comparison with that in LFS SOL. However, there has been little observation on electrostatic fluctuation in HFS SOL so far. It becomes one of the most important issues to reveal an influence of plasma blobs on cross-field transport in the HFS SOL. Furthermore, the analysis of the fluctuation property is required to understand the generation mechanism of plasma blobs and to predict the cross-field transport, including non-locality of transport in the edge plasma turbulence.

In this proceeding, detailed comparison between fluctuation characteristics at HFS and LFS SOLs in addition to near $\mathrm{X}$ point will be made in the $\mathrm{L}$ mode plasma using reciprocating Langumuir probes[10]. Statistical analysis based on probability distribution function (PDF) is employed to describe intermittent (non-diffusion) transport in SOL plasma fluctuations[11]. 

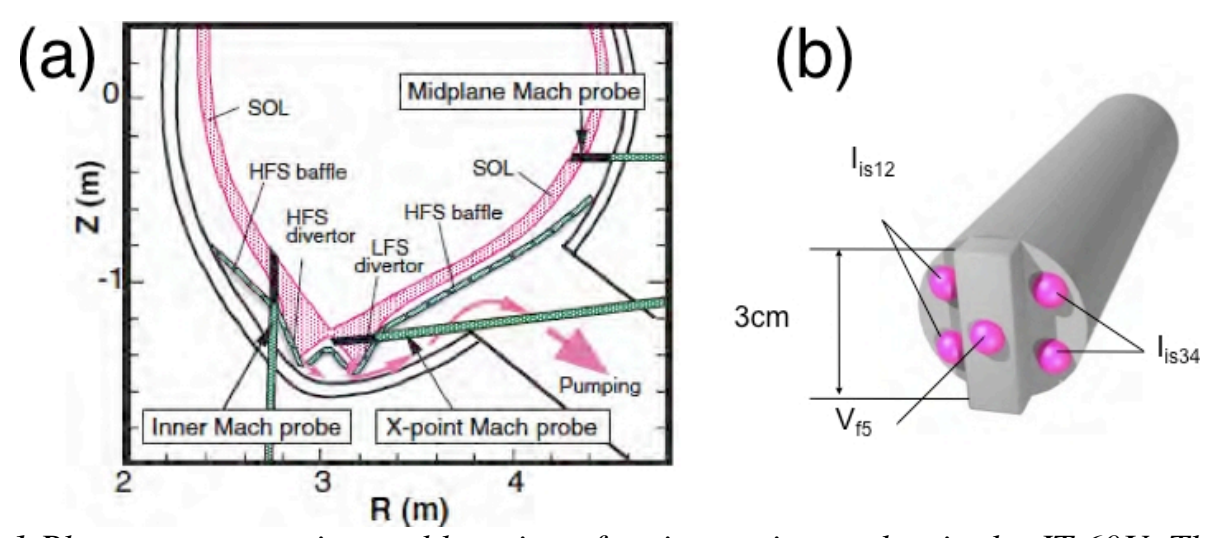

Fig. 1 Plasma cross-section and location of reciprocating probes in the JT-60U. Three reciprocating Mach probes measure radial profiles of SOL plasma at LFS midplane, $X$ point and above HFS baffle.

\section{Experimental Setup}

In the JT-60U, three reciprocating Mach probes are installed at LFS mid-plane, HFS and just below the X-point as shown in Fig. 1(a). The fluctuation property was investigated from fast sampling $(500 \mathrm{kHz})$ signals of the ion saturation current $\left(j_{s}\right)$ measured at LFS midplane, $X$ point and above HFS baffle. Each reciprocating probe has 5 electrodes as shown in Fig. 1(b). 4 electrodes are used as double probe to measure $j_{s}$. Floating potential $V_{\mathrm{f}}$ is also measured with the electrode at the top.

\section{Poloidal dependence of fluctuation characteristics}

\subsection{Statistical analysis based on probability distribution function}

Fluctuations of ion saturation currents js were measured at three poloidal locations in L-mode plasmas (shot number \#45723 for HFS SOL and LFS SOL, shot number \#48473 for X-point as reference).

Figure 2 shows radial profiles of the averaged ion saturation currents $\left\langle j_{s}\right\rangle$ and fluctuation level $\left(\delta j_{s} /<j_{s}>\right) . \Delta r$ is distance form the separatrix at LFS midplane. The probe position at HFS SOL and X-point are mapped to the LFS midplane by tracing the magnetic field line. $\delta j_{s} /<j_{s}>$ is gradually increased at the LFS SOL, while at HFS SOL, $\left.\delta j_{s} /<j_{s}\right\rangle$ is small less than $10 \%$ and decreased with $\Delta r$. Figure 3 shows the PDFs of $j_{s}$ at HFS and LFS SOLs, and X-point, which indicate that the PDF at LFS SOL is skewed positively,

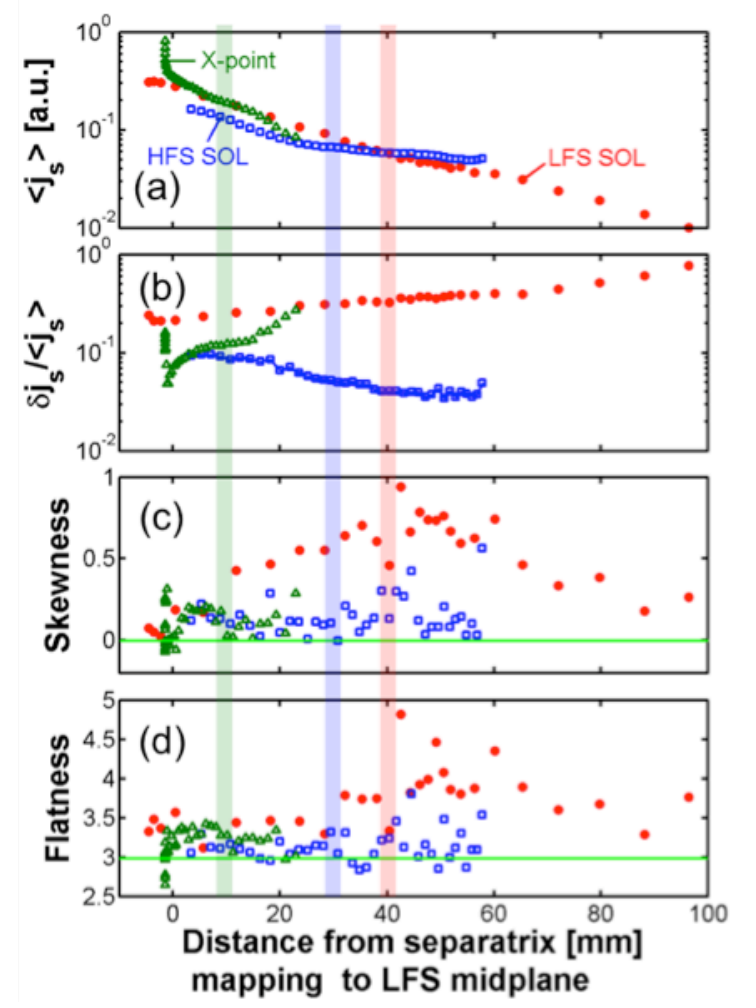

Fig.2 (a)averaged $j_{s}$ profiles measured with LFS midplane (circles) and HFS (squares) Mach probes in L-mode. (b) fluctuation level of $j_{s},(c)$ skewness. 
suggesting non-diffusive plasma transport (blobby transport) frequently occurs at LFS midplane. On the other hand, the PDF at HFS SOL is close to Gaussian distribution with less coherent events.

Skewness and flatness are defined by the $3^{\text {rd }}$ and $4^{\text {th }}$ moments of PDF, normalized by the standard deviation, i.e. $S=\left\langle x^{3}\right\rangle \mid\left\langle x^{2}\right\rangle^{3 / 2}$ and $F=\left\langle x^{4}\right\rangle \mid\left\langle x^{2}\right\rangle^{2}$, respectively. Profile of PDF can be characterized by skewness and flatness. When the PDF is Gaussian distribution, $S$ and $F$ become 0 and 3, respectively. When large positive bursts occur in the fluctuation signal, PDF is positively skewed and becomes flatten compared to Gaussian distribution, leading to $S>0$ and $F>3$. Fig. 2(c) shows skewness of the PDF at LFS SOL becomes maximum around $\Delta r=5 \mathrm{~cm}$ and is positive value in wide radii $(\Delta r<10 \mathrm{~cm})$. On the other hand, skewness and flatness of the PDFs at HFS SOL are close to 0 and 3 in wide radii, meaning the PDFs at HFS SOL obey Gaussian statistics with random process.

In order to analyze spatial change of PDFs at LFS SOL more quantitatively, we employ Kullback-Leibler (K-L) divergence[12].

For probability distributions $P\left(s_{i}\right)$ and $Q\left(s_{i}\right)$ of a discrete random variable $s_{i}=\left\{s_{1}, s_{2}, \cdots, s_{n}\right\}$, the $\mathrm{K}-\mathrm{L}$ divergence $D(P \| Q)$ of $Q\left(s_{i}\right)$ from $P\left(s_{i}\right)$ is defined to be

$$
D(P \| Q)=\sum_{\left\{s_{i}\right\}} P\left(s_{i}\right) \log _{e}\left(P\left(s_{i}\right) / Q\left(s_{i}\right)\right) .
$$

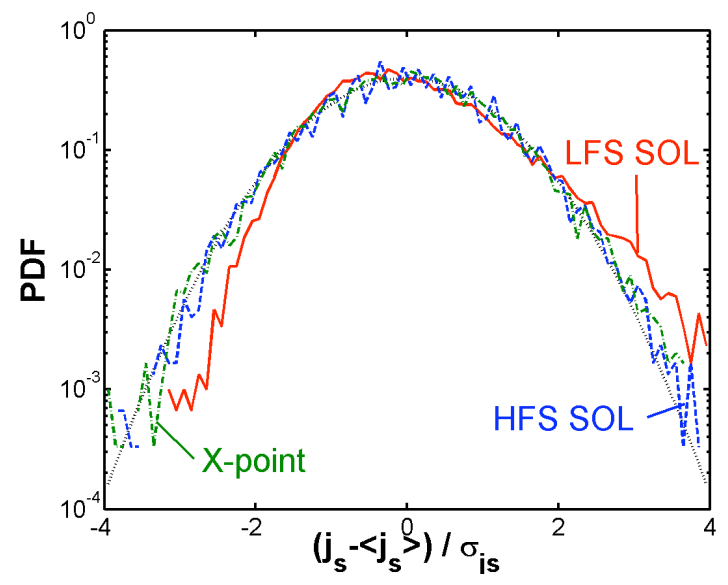

Fig. 3 Log-linear plots of probability distribution function (PDF) of $j_{s}$ measured at LFS and HFS SOLs, and $X$-point.

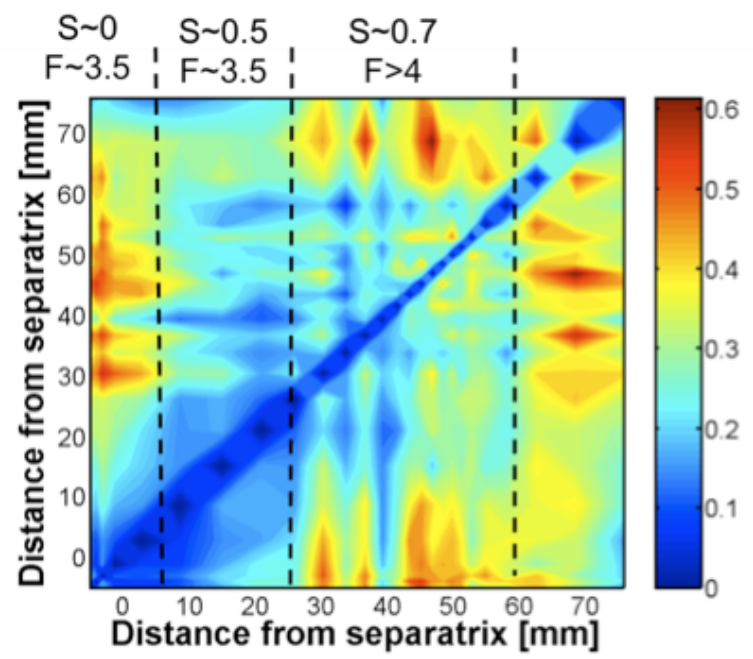

From the definition of $D(P \| Q)$, $D(P \| Q)$ cannot be negative value.

Fig. 4 Kullback-Leibler divergence of PDFs at difference radii in $L F S S O L$.

Only if $P\left(s_{i}\right)=Q\left(s_{i}\right), \quad D(P \| Q)$

becomes zero. Then, K-L divergence is index to represent the similarity of two probability distribution functions.

Figure 4 shows the K-L divergence calculated from PDFs at different radii in the LFS SOL. In radii $(0<\Delta r<2.5 \mathrm{~cm})$, it is found that PDFs are not changed so much, probably because diffusive plasma transport associated with random process is dominating near the separatrix. From $\Delta r=2.5 \mathrm{~cm}$, PDFs are gradually changed because K-L divergence becomes positive. Above $\Delta r=6.0 \mathrm{~cm}$, PDFs are dramatically modified in comparison with PDFs at radii $\Delta r<6.0 \mathrm{~cm}$. In the previous study, Mach probe measurement shows direction of the poloidal plasma flow changes around $\Delta r=6.0 \mathrm{~cm}$. This results could be associated with the change of PDFs at $\Delta r=6.0 \mathrm{~cm}$ shown in Fig. 4. 


\section{2 Fourier analysis and auto-correlation function}

The scaling features of the fluctuation can be studied by mean of Fourier analysis. Figure 5 shows the spectral density of $j_{s}$, at HFS and LFS SOLs, and X-point. All $S(f)$ have no peaks in $S(f)$ at frequencies less than $30 \mathrm{kHz}$. Above $30 \mathrm{kHz}$, there are some peaks in $S(f)$. These peaks could be due to noise generated by the pre-amplifier in the probe measurement circuit damaged by neutron irradiation, because the replacement of the pre-amplifier can reduce the noise components dramatically in high frequency region.

The shapes of the spectral density $S(f)$ allow one to conclude whether the scaling behavior of a time series can be described by power-law dependences of the type $S(f)$ $=f^{-\alpha}$. The power spectrum of the turbulent fluctuations quantifies the properties of the process. A self-organized criticality (SOC) model was proposed to analyze non-local plasma transport in tokamaks $[13,14]$, which predicts a power law dependence, $\quad S(f)=$ $f^{-1}$. In the LFS SOL, scaling sub-range with respect to the frequency is clearly observed in Fig. 5. Typical value of the scaling exponent $\alpha$ of the power spectra in the scaling sub-range is 1.3 , which is different from the SOC prediction.

Figure 6 shows auto correlation function

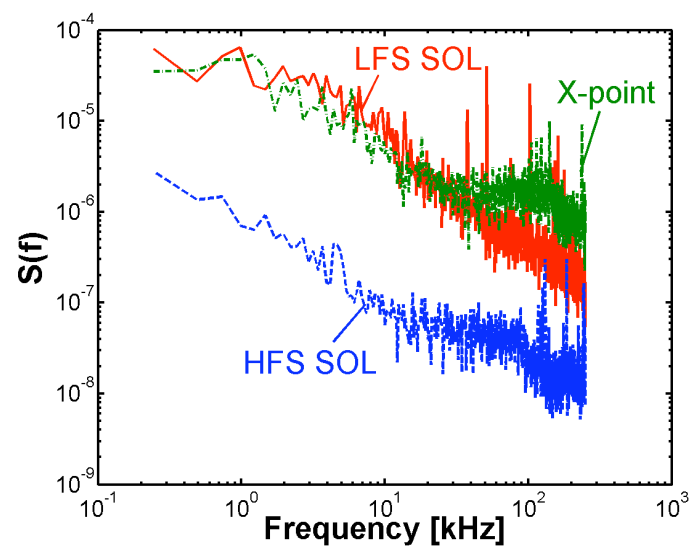

Fig. 5 Power spectra of $j_{s}$ at LFS and HFS

SOLs and X-point.

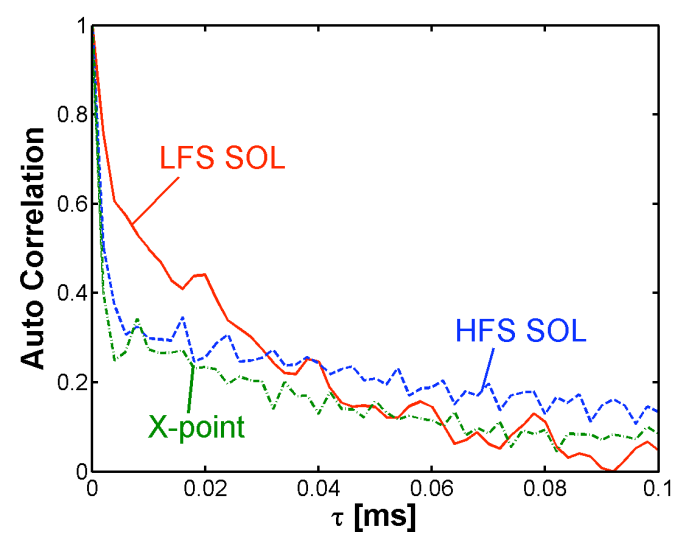

Fig. 6 Auto correlation function of $j_{s}$ at LFS and HFS SOLs and X-point. of $j_{s}$. It is found that auto correlation time of $j_{s}$ in HFS SOL and X point is quite short, meaning the fluctuation signals in HFS SOL and X point are randomized. On the other hand, the auto correlation time at LFS SOL is relatively large, indicating Levy type process.

\section{3 Waiting and duration time statistics}

We have analyzed statistics of waiting-time and duration-time of $j_{s}$ with positive burst events, where a positive burst is defined by a positive spike with an amplitude above twice of the standard deviation of the original signal. In the statistics of duration-time as shown in Fig. 7(b), it is found, for the first time, that the PDF for the duration time $\tau$ clearly shows a statistical self-similarity fluctuation property at LFS SOL, because the logarithmic plot of the PDF gives the power factor scaling $P(\tau) \sim \tau^{-\beta}$. Such a power factor scaling is sometime observed in explosive phenomena. In term of chaotic analysis, $\beta$ corresponds to a capacity fractal dimension. 
At HFS SOL and X-point, the PDF for the duration time $\tau$ looks like an exponential law: $P(\tau)$ $\sim \exp (-\tau)$. Moreover, waiting time statistics of $j_{s}$ in Fig. 7 (a) also shows same tendency although the PDF for the waiting time is rather scattered. As mentioned before, a selforganized criticality (SOC) model was employed to analyze non-local plasma transport like plasma blobs in tokamaks[13]. The SOC model predicts that the PDFs of the waiting times and duration times obey an exponential law: $P(\tau) \sim \exp (-\tau)$. However, the PDFs at LFS-SOL
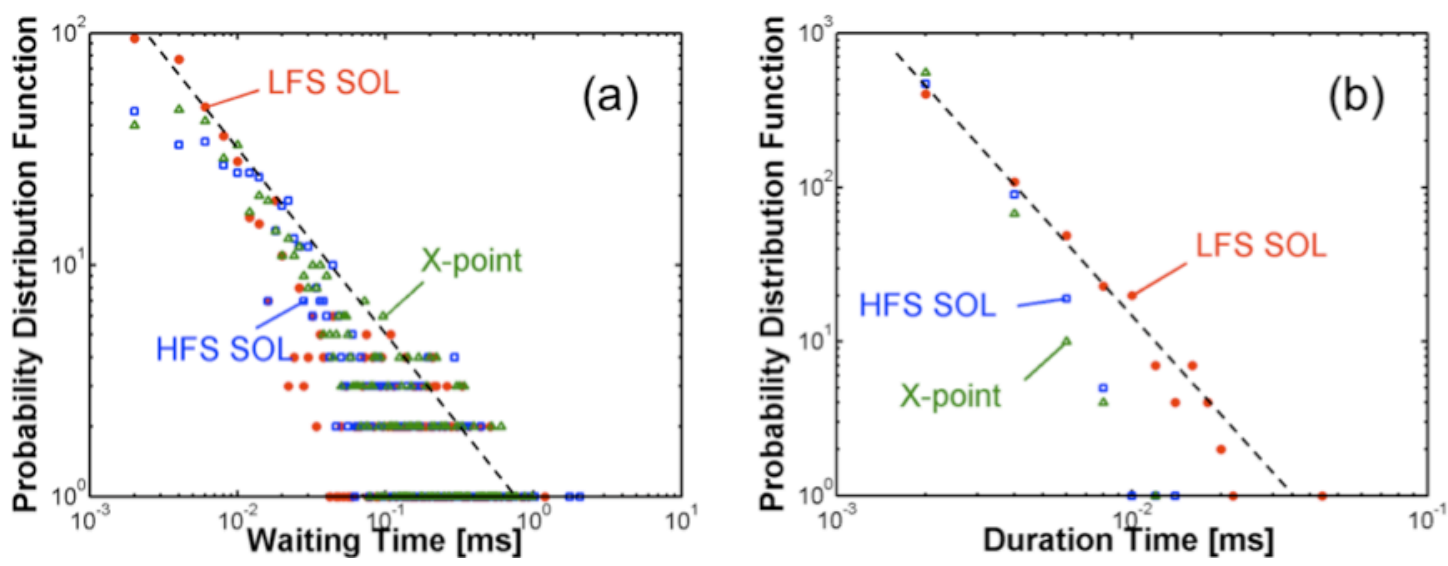

Fig. 7 Probability distribution function of (a) the waiting time and (b) duration time of bursts. Power low fits are shown by solid and dashed lines.

shows the power factor scaling: $P(\tau) \sim \tau^{-\beta}$, which is different from the SOC prediction. Thus, the waiting time and duration time statistic at LFS-SOL are in contrast with the prediction of the SOC model, which are consistent with the power law of the power spectrum mentioned before. These experimental results are similar ones observed in the RFP plasma[15]. On the other hand, when the fluctuation signal obeys Poisson process, PDFs for waiting time and duration time also gives an exponential law: $P(\tau) \sim \exp (-\tau)$. Then, the exponential law of the PDFs in HFS-SOL and X-point means that the fluctuation property is fully random, which could be related to the fact that PDFs of $j_{s}$ at HFS-SOL and X-point are very close to Gaussian distribution as shown in Fig. 3.

\section{Analysis of positive burst's profile}

In order to reveal the typical burst's profile at LFS SOL, conditional averaging method was employed. In this method, large bursts of $j_{s}$ with a peak above three times as large as the standard deviation of the original signal are selected and averaged in the same time domain. The result indicates that the positive spikes have the common property of a rapid increase and slow decay shown in Fig. 8. This feature is similar to that of theoretical prediction for plasma blobs. The conditional average of $V_{f}$ measured simultaneously using a probe tip 5 $\mathrm{mm}$ inside of the probe tip for $j_{s}$ shows that $V_{f}$ changes from positive to negative with

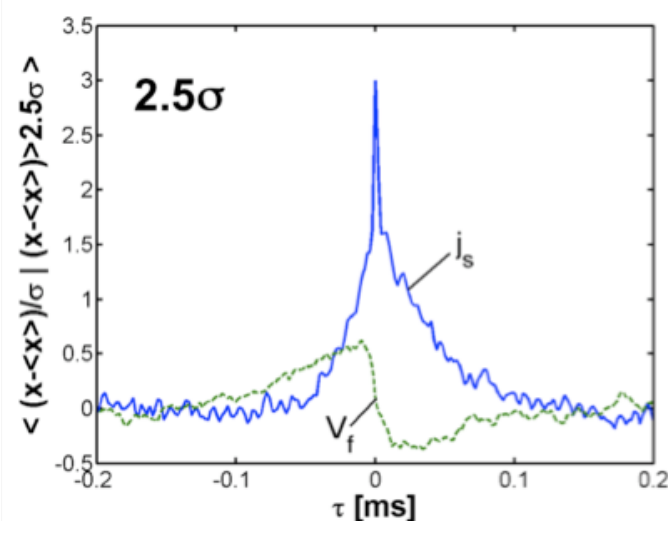

Fig. 8 Conditional averaging results of $j_{s}$ and floating potential $V_{f}$ at LFS SOL. 
respect to $\left\langle V_{f}\right\rangle$ as a large burst of $j_{s}$ passes by. This result gives the internal potential structure of plasma blobs. The plasma blob velocity also can be estimated by using delayed time between the peaks of $j_{s}$ and $V_{f}$ to be about $425 \mathrm{~m} / \mathrm{s}$.

Although the conditional averaging method extracts the property of positive bursts as shown in Fig. 9, careful comparison between the Fig. 9 and original signal shown in Fig. 10 (a) shows the reconstructed signal in Fig. 9 is broader profile, especially at ramp-up phase of the positive burst. In order to reveal the property of positive bursts more precisely, we employ conditional averages with VITA ( Variable Interval Time Average) method[16], which allow to detect bursty events associated with the sudden variations of the signal in the time domain.

The localized variance $v_{\mathrm{a}}$ is defined by

$$
v_{a}(t)=\frac{1}{T} \int_{t-T / 2}^{t+T / 2}\left(j_{s}-\left\langle j_{s}\right\rangle\right)^{2} d t^{\prime} .
$$

The detection function $a_{\mathrm{v}}$ is also defined by using the localized variance $v_{\mathrm{a}}$ as follows:

$$
a_{v}(t)= \begin{cases}1 ; & v_{a}(t) \geq k\left\langle\left(j_{s}-\left\langle j_{s}\right\rangle\right)^{2}\right\rangle, \\ 0 ; & \text { else }\end{cases}
$$

where $k$ is set to be 2 in this analysis and averaging time window is $24 \mu \mathrm{sec}$. The averaged burst signal is obtained with conditional averages by using the detection function $a_{\mathrm{v}}$.

Figure 10 shows the positive burst profile reconstructed by the conditional averages with the VITA method. It is found that the VITA method can provide the burst profile with faster ramp-up. The VITA method is very the positive bursts in the signal.

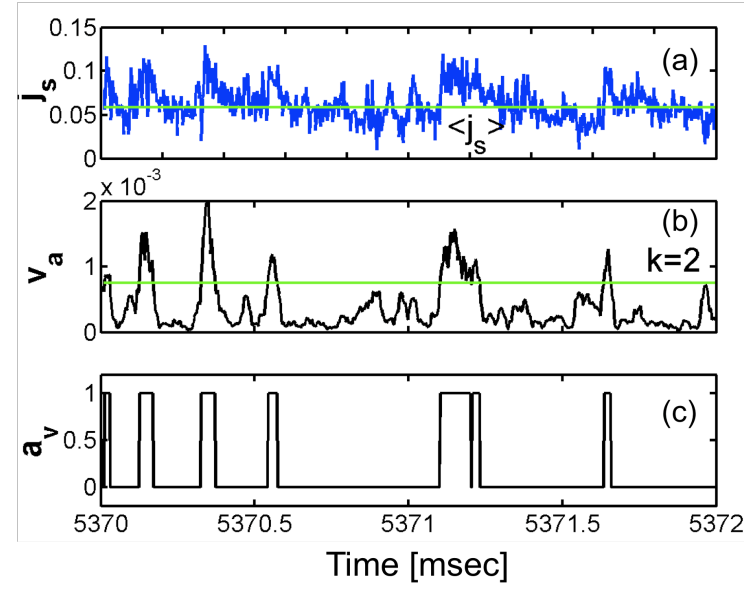

Fig. 9 (a) time evolution of ion saturation current $j_{s}$ at LFS SOL, (b) the localized variance $v_{\mathrm{a}}$ and $(c)$ detection function $a_{\mathrm{v}}$.

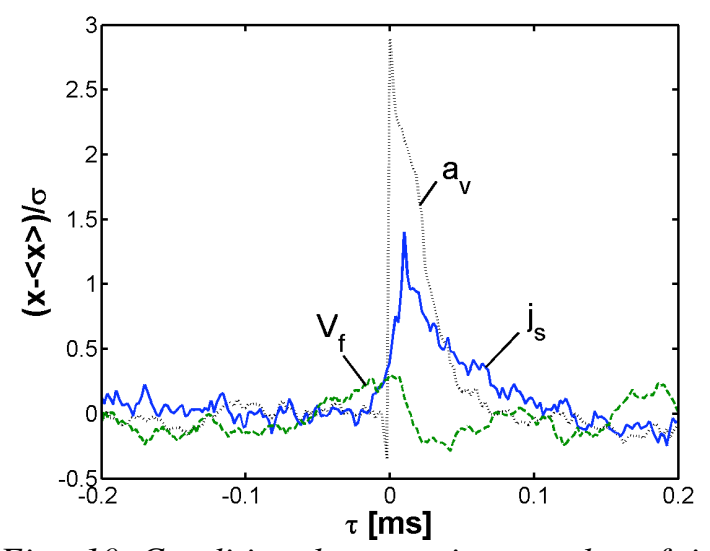

Fig. 10 Conditional averaging results of $j_{s}$ and floating potential $V_{f}$ at LFS SOL with VITA method

\section{Conclusion}

We have done a detailed comparison between fluctuation characteristics at HFS and LFS SOLs, and X-point in the L mode plasma of JT-60U tokamak using reciprocating Langumuir probes. Statistical analysis shows intermittent transport in SOL plasma fluctuations. It was found that the positive bursty events appeared most frequently at LFS midplane, then the PDF is strongly skewed positively, while the PDF in HFS SOL is close to Gaussian distribution. Conditional averaging analysis of the positive bursty events at LFS midplane indicates the intermittent feature with a rapid increase and a slow decay is similar to that of plasma blobs theoretically predicted. Statistical self-similarity was also investigated. It was found that clear statistical self-similarity was observed at LFS SOL, showing fractal property of the fluctuation. 


\section{Acknowledgments}

One of authors (N. O.) wishes to thank Mr. Takagi for his excellent technical supports. This work is partially supported by NIFS/NINS under the project of Formation of International Network for Scientific Collaborations.

\section{References}

[1] ZWEBEM S.J., et al., Nucl.Fusion 23 (1983) 825.

[2] MOYER R.A., et al., Plasma Phys. Control. Fusion 38 (1996) 1273.

[3] BUDAEV V., et al., Plasma Phys. Control. Fusion 3 (1993) 429.

[4] ANTAR G.Y. et al., Phys. Rev. Lett. 87 (2001) 065001.

[5] CARRERAS B.A. et al., Phys. Plasmas 8 (2001) 3702.

[6] TERRY J.L. et al., Phys. Plasmas 10 (2003) 1739.

[7] BOEDO J. A. , et. al., Phys. Plasmas, 10 (2003) 1670.

[8] BUDAEV V., et. al., Nucl. Fusion, 46, (2006) S181.

[9]KRASHENINNIKOV S., Phys. Lett. A 283(2001) 368.

[10] ASAKURA N. , et. al., Nucl. Fusion 44 (2004) 503-512.

[11]MIYOSHI H., et. al. 31 st EPS Plasma Physics Conference, London (2004) P5.101.

[12] KULLBACK S. and LEIBLER R. A. (1951) On information and sufficiency, Annals of Mathematical Statistics 22:79-86.

[13] DIAMOND P.H., et. al., Phys. Plasma 2 (1995) 3640.

[14] POLIZER, P. A., et. al., Phys. Rev. Lett. 84 (2000) 1192.

[15] SPADA E., et. al., Phys. Rev. Lett. 86 (2001) 3032.

[16]BLACKWELDER, R. F. and KAPLAN, R. E., J. Fluid Mech., 76-1(1976)89. 